\title{
Nesfatin-1, a unique regulatory neuropeptide of the brain
}

\author{
Artur Pałasz ${ }^{\mathrm{a}}$, Marek Krzystanek ${ }^{\mathrm{b}}$, John Worthington ${ }^{\mathrm{c}}$, Beata Czajkowska ${ }^{\mathrm{c}}$, \\ Karol Kostro ${ }^{d}$, Ryszard Wiaderkiewicz ${ }^{\text {a }}$, Grzegorz Bajor ${ }^{d}$
}

${ }^{a}$ Department of Histology, Medical University of Silesia

Medyków Street 18, 40-752, Katowice, Poland

${ }^{\mathrm{b}}$ Department and Clinic of Psychiatry and Psychotherapy, Medical University of Silesia Ziolowa Street 45/47, 40-635, Katowice, Poland

${ }^{c}$ Immunology Group, Faculty of Life Sciences, University of Manchester AV Hill Building, Oxford Road, Manchester M13 9PT, UK

${ }^{\mathrm{c}}$ Department of Anatomy, Medical University of Silesia, Medyków Street 18, 40-752, Katowice, Poland

Corresponding author;

A. Pałasz PhD; Department of Histology, Medical University of Silesia, ul. Medyków 18, Katowice 40-752, Poland. e-mail: apalasz@ sum.edu.pl 


\begin{abstract}
Nesfatin-1, a newly discovered NUCB2-derived satiety neuropeptide is expressed in several neurons of forebrain, hindbrain, brainstem and spinal cord. This novel anorexigenic substance seems to play an important role in hypothalamic pathways regulating food intake and energy homeostasis. Nesfatin-1 immunoreactive cells are detectable in arcuate (ARC), paraventricular (PVN) and supraoptic nuclei (SON), where the peptide is colocalized with POMC/CART, NPY, oxytocin and vasopressin. The nesfatin-1 molecule interacts with a G-protein coupled receptor and its cytophysiological effect depends on inhibitory hyperpolarization of NPY/AgRP neurons in ARC and melanocortin signaling in PVN. Administration of nesfatin-1 significantly inhibits consumatory behaviour and decreases weight gain in experimental animals. These recent findings suggest the evidence for nesfatin-1 involvement in other important brain functions such as reproduction, sleep, cognition and anxiety- or stress-related responses. The neuroprotective and antiapoptotic properties of nesfatin-1 were also reported. From the clinical viewpoint it should be noteworthy, that the serum concentration of nesfatin-1 may be a sensitive marker of epileptic seizures. However, the details of nesfatin-1 physiology ought to be clarified, and it may be considered suitable in the future, as a potential drug in the pharmacotherapy of obesity, especially in patients treated with antipsychotics and antidepressants. On the other hand, some putative nesfatin-1 antagonists may improve eating disorders.
\end{abstract}

Key words; nesfatin-1, NUCB2, neuropeptides, hypothalamus, anorexia 


\section{Contents}

1. Introduction

1.1. Overview

1.2. Molecular structure

1.3. Distribution

2. Anorexigenic activity of nesfatin-1 at the level of hypothalamus

2.1. Mode of action in the arcuate nucleus

2.2. Interactions with oxytocin, $\mathrm{CRF}$ and melanocortin pathway

3. Involvement of nesfatin-1 in the reproductive processes

4. Neuropsychiatric aspects of nesfatin-1 physiological actions

4.1. Stress responses and depression

4.2. Eating disorders

4.3. Epilepsy

5. Concluding remarks

6. References 


\section{Introduction}

\subsection{Overview}

In recent years, thanks to a dynamic development of molecular biology, a number of new regulatory neuropeptides have been identified and described. A great majority of them have unique characteristics, a wide multidirectional spectrum of physiological activity and act at the level of many neuronal pathways. In this context, a recently discovered and still relatively unknown nesfatin-1 (NEFA/NUCB2-encoded satiety and fat-influencing protein) appears to be a particularly interesting substance. The first information regarding its existence was provided in 2006 by Oh et al., as result of studies on lung carcinoma cell lines, revealing the expression of leptin receptors following activation by the PPAR- $\gamma$ ligand - troglitazone. Presently, research studies on nesfatin-1 and its neurophysiological properties are still in their initial phase.

Nesfatin-1 is a potent anorexigenic factor inducing satiety and strongly inhibiting food and water intake (Stengel and Tache 2010, Stengel et al. 2010a, Pałasz et al. 2010, Shimizu et al. 2009). Upon direct injection to a lateral ventricle of the rat brain, it causes a dose dependent depression of consumatory behaviour. Continuous infusion to the IIIrd ventricle results in a significant reduction of body mass and in a decreased amount of white adipose tissue. An intraperitoneal injection of nesfatin-1 induces a 3 - hour suppression of food intake in mice. Also, its subcutaneous administration induces the identical effect, and this anorexigenic action can be maintained for 14 hours. Repeated intraperitoneal doses have substantially inhibited the increase of body mass, over a 6day period (Shimizu et al. 2009). It should be underlined that the peripheral nesfatin-1 doses required to suppress food intake are approximately 1000-fold higher than those effective in the CNS (Stengel et al. 2010a). 
Serum levels of nesfatin-1 are substantially decreased in the state of starvation, while refeeding leads to normalization. Nesfatin-1 penetrates the blood-brain barrier (Pan et al. 2007) that may potentially create an opportunity for its putative therapeutic use. It appears that after reaching the hypothalamic centres, nesfatin-1 may inhibit appetite and food intake. It has recently been noted that in humans, the CSF/plasma nesfatin-1 ratio is negatively correlated with body mass index (BMI) and body mass, possibly suggesting that nesfatin-1 is a protein-bound neuropeptide. A hypothesis has been proposed that, dependent on body mass changes, efficiency of nesfatin-1 uptake by the CSF can be caused by saturation of its transporters (Tan et al. 2011). Recently, studies clearly suggesting the participation of nesfatin-1 in brain actions diverse from energy balance regulation have been published. Nesfatin-1 appears to be a neuropeptide that plays a significant role in reproductive processes, stress responses, and pathology of mental and neurological disorders (Könczöl et al. 2010, Ogiso et al. 2011, Aydin et al. 2011, Stengel and Tache 2011). These completely innovative aspects of nesfatin-1 physiology are more extensively presented in subsections of this review.

With regard to the anorectic properties of nesfatin-1, the recent presumption that it can display some characteristics of neuroprotective factors seems particularly intriguing. At the present time, the only source of these suggestions is the study conducted by Özsavci et al., in 2010, who examined the influence of nesfatin-1, administered intraperitoneally, on the profile of oxidative stress markers and the permeability of blood-brain barrier in rats with subarachnoid hemorrhage (SAH). The authors have noted that the levels of SAH-induced oxidative brain injury markers and plasma levels of pro-inflammatory cytokines: TNF- $\alpha, \mathrm{IL}-1 \beta$, and IL- 6 as well as proapoptotic protein caspase-3 were significantly decreased in animals exposed to the actions of nesfatin-1. Moreover, these authors have reported that nesfatin- 1 reduced the, SAH-dependent, histological structural changes of basilar arteries by inhibiting neutrophil infiltration. This research allows us to deduce that nesfatin-1 can play the role of anti-inflammatory and antiapoptotic factor in the central nervous system. However, as of today, it is still too early to conclude that nesfatin-1 represents a compound which plays the key role in 
the neuroprotective mechanisms of the brain. There is no doubt that this remarkably interesting issue requires further experimental studies.

\subsection{Molecular structure}

Formation of the 82 -amino acid molecule nesfatin-1 is the effect of posttranslational cleavage of prohormone NEFA/nucleobindin-2 (NUCB2), performed by the specific convertases PC3/1 and PC2 (Garcia-Galiano et al. 2010, Stengel and Tache 2010). NUCB2, a polypeptide composed of 396 amino acids (aa), preceded by a 24-aa signal peptide is located both on the plasma membrane and in the neuroplasm. This precursor protein consists of the following domains: $\mathrm{N}$-terminal signal peptide, Leu/lle rich region, DNA-binding domain, nuclear targeting signal, two $\mathrm{Ca}^{2+}$-EF-hand motifs and leucine zipper domain (Stengel et al 2010a). Nesfatin-1 has a substantial, above $85 \%$ homology, of its sequences between humans and mammals, and even the lower vertebrates (Gonzales et al. 2010). The nesfatin-1 molecule is composed of 3 domains: $\mathrm{N}$-terminal (N23), middle part (M30) and C-terminal (C29). The M30 active core appears to play the key role in the induction of physiological effects of this peptide, and especially in its anorectic responses (Fig1. A). Another effect of the NUCB2 proteolytic processing is a production of its inactive derivatives: nesfatin 2 and 3, spanning residues 85-163 and 166-396, respectively (Oh et al. 2006). 


\subsection{Distribution}

In the rat hypothalamus, neurons localized in the arcuate nucleus (ARC), paraventricular nucleus (PVN), supraoptic nucleus (SON), dorsomedial (DMH), lateral hypothalamus (LHA), and zona incerta are characterized by expression of nesfatin-1 (Stengel et al. 2010a, Shimizu et al. 2009). It is assumed that the anorexigenic action of nesfatin-1 is performed mostly in the first three key regulatory hypothalamic centres. The nesfatin-1 immunopositive neurons are also present in the brain stem, including serotoninergic cells - of the raphe pallidus (RPa), raphe obscurus (ROb), and cholinergic cells - of the nucleus accessorius of oculomotor nerve (nucleus Westphal-Edinger; EW), and nucleus dorsalis of vagus nerve (Foo et al. 2008). Since some sympathetic axons originate from the raphe nuclei and terminate in brown adipose cells, it is suggested that nesfatin-1 takes part in the regulation of thermogenesis (Brailoiu et al. 2007). It is also possible that nesfatin-1, which is released from the synaptic endndings' of the vagus nerve, can have an impact on secretory and motor activity of the gastrointestinal tract, and may regulate the course of digestive functions (Stengel et al. 2010a, Goebel et al. 2009). On the other hand, it has recently been demonstrated that pretreatment with capsaicin (to block autonomic C fibres) abolished the food intake reduction caused by peripheral nesfatin-1 injection. These findings indicate a putative role of vagal afferents in peripheral nesfatin-1 signaling to the brain centres (Shimizu et al. 2009). As further evidence to this, it has been demonstrated that nesfatin-1 activates cell bodies in the nodose ganglion in vitro. This assembly of neurons contains the perikarya of vagal nerve fibres projecting to the nucleus of the solitary tract (Iwasaki et al. 2009). The nesfatin-1 contribution to the physiological functions of the oculomotor nerve has not been clearly explained (Goebel et al. 2009). In addition, perikarya of the piriform, insular and cingulate cortex, endopiriform nucleus, lateral septum, anterior cortical and central amygdaloid nucleus, bed nucleus of stria terminalis and interstitial nucleus of the posterior limb of anterior commissure, are also characterized by nesfatin-1 immunoreactivity. The neuropeptide was also identified in other structures of brain stem, in nucleus of the solitary tract (NST), in dorsal raphe nucleus, raphe magnus 
nucleus, gigantocellular reticular nucleus, lateral parabrachial nucleus, nucleus ambiguous, central gray (nucleus O), and even in the Purkyne's cells of cerebellar cortex. Moreover, the presence of nesfatin-1 was detected in sympathetic and parasympathetic preganglionic neurons, originating from thoracic, lumbar and sacral segments of the spinal cord (Goebel et al. 2009). One of the achievements of new experimental research was the discovery of nesfatin-1 in those regions of animal brain in which it was not previously identified. Although the distribution of nesfatin-1 in the mice brain is in general very similar to the one observed in rat, many other previously unknown areas in which this peptide is encountered including: anterior olfactory nucleus (AOL), accumbens nucleus (Ac), caudate putamen, piramidal cell layer of the hippocampus, dorsal and dorsolateral tegmental nuclei, nucleus of the facial nerve, area postrema, inferior olivary nucleus, and a newly identified region, called intermediate dorsomedial hypothalamus (IDMH) were found in these species (Goebel-Stengel et al. 2011). Distribution of nesfatin-1 in perikarya of autonomic brain centres, preganglionic visceral motoneurons of spinal cord, and forebrain nuclei involved in pain perception and in cognitive processes, seems to confirm a presumption that the physiological role of this neuropeptide is far beyond the sphere of food-intake regulation. In particular, it participates in vasomotor, neurosecretory, and emotional functions.

Although, the present article emphasizes the nesfatin-1 activity in some brain structures, the fact that the expression of this peptide has also been revealed in cells of some other organs cannot be neglected. In particular, nesfatin-1 has been identified in gastric glands, in submucosal layer of the duodenum (Zhang et al. 2010), and in B cells of the pancreatic islets (Foo et al. 2010). It should be mentioned, that in the gastric glands, the majority of nesfatin-1 immunopositive X/A-like endocrine cells are characterized by coexpression of ghrelin, an orexigenic hormone (Stengel et al. 2010), and only a small number of these cells reveals the presence of somatostatin (Maejima et al. 2009, Stengel et al. 2009). The newest reports suggest that in vitro, nesfatin-1 augments the glucose-induced insulin secretion through the activation of L-type calcium channels in the B cells of mice (Nakata et al. 2011 Gonzalez et al. 2011). In turn, an elevated glucose 
level increases significantly the nesfatin-1 release by pancreatic endocrine cells (Foo et al. 2010). It has been reported that the administration of nesfatin-1 reduces a blood glucose level in mice with a hyperglycemic genetic profile (Su et al. 2010). Expression of NUCB2 and nesfatin-1 has also been revealed in human and murine adipocytes. It is noteworthy that this expression is particularly evident in the subcutaneous fat tissue cells (Ramajaneya et al. 2010). Recently, some preliminary reports regarding identification of the nesfatin-1 immunopositive cells in testis and ovaries of rat have appeared in the literature. (Garcia-Galiano et al. 2010a).

\section{Anorexigenic activity of nesfatin-1 at the level of hypothalamus}

\subsection{Mode of action in the arcuate nucleus}

The arcuate nucleus (ARC), which is excluded from the blood-brain barrier, is one of the hypothalamic structures participating in energy balance regulation. It's neurons are characterized by a particular sensitivity to peripheral hormonal signals such as circulating blood molecules of adipokines, ghrelin, insulin, cholecystokinin (CCK), peptide YY (PYY), oxyntomodulin, and some other compounds. A structure of ARC consists of two functionally antagonistic groups of neurons. The first group is composed of cells, which synthesize orexigenic factors - neuropeptide Y (NPY) and Agouti-related protein (AgRP). The second group contains neurons producing pro-opiomelanocortin (POMC), which has active derivatives, including a-MSH and cocaine-amphetamine regulated transcript (CART), characterized by anorexigenic properties. It was originally suggested that only the POMC/CART neurons of the rat hypothalamus are characterized by nesfatin-1 expression, and that in the NPY/AgRP cells this peptide has not been identified. However, some later studies have found that the relatively limited population of NPY cells (approximately 20\%) reveals immunoreactivity of nesfatin-1 that to some degree complicates an explanation of the some signaling events in ARC, related to this 
peptide (Inhoff et al. 2010). A more detailed analysis of coexpression of the nesfatin ARC (Fig 2.) profile has revealed that above $60 \%$ of $A R C$ cells synthesize POMC and CART, $46 \%$ - NPY, 29\% - growth hormone-releasing hormone (GHRH), and 14\% - neurotensin (Stengel et al. 2010a). Nesfatin-1, which is released from the POMC/CART neurons, directly inhibits the NPY/AgRP neurons, causing their hyperpolarization, through the

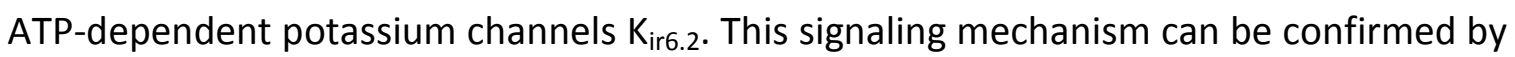
an observation that glibenclamide, an antagonist of the same type of $\mathrm{K}^{+}$channels, relieves this peptide's physiological effect. There are some suggestions that the hyperpolarization of orexigenic ARC neurons can be a key phenomenon in anorexia, induced by nesfatin-1 (Price et al. 2008). However, according to other reports, nesfatin1 contributes to an increase of $\mathrm{Ca}^{2+}$ ion concentration in neuroplasma of the target cells. This can be reversed by using pertussis toxin, clearly suggesting that this neuropeptide is also a ligand of the metabotropic receptor, connected with $\mathrm{G}_{\mathrm{i} / \mathrm{o}}$. protein (Fig 1. B). It has been found that the receptors activation leads to opening of the $L$ and $P / Q$-type calcium channels, since the use of selective inhibitors of these channels such as verapamil and $\omega$-conotoxin results in removal of the nesfatin-1 dependent influx of $\mathrm{Ca}^{2+}$ ions (Brailoiu et al. 2007).

Interestingly enough, the nesfatin-1 induced elevation of $\mathrm{Ca}^{2+}$ ion concentration is also reduced by KT5720, the specific inhibitor of protein kinase $A$, suggesting a contribution of this CAMP - dependent enzyme in the signal transduction process. This is a very unusual phenomenon, since the effect of $G_{i}$ protein activation is ex definition the inhibition of adenyl cyclase, causing a decrease of the cAMP concentration, and in consequence, inhibition of protein kinase A. One possible explanation of this surprising issue is the suggestion that nesfatin-1 is able to activate some different signaling pathways, influencing cross-binding of the receptor with various types of G protein, initially by activating $G_{i}$, and then, by stimulating $G_{s}$ (Brailoiu et al. 2007). Unfortunately, the nesfatin-1 receptor has not been cloned yet. This makes conducting of the detailed pharmacological studies impossible. It also causes some serious difficulties in searching for molecules which can be potential modulators. It should be underlined that nesfatin- 
1 is not released from presynaptic axons, but it is secreted by dendrites, and acts as a local paracrine or/and autocrine factor (Yosten and Samson 2010). Nesfatin-1 may also be involved in deacylation of ghrelin, administered peripherally, which relieves ghrelin's orexigenic action among rats. These actions have been revealed in experimental rat studies, in which the nesfatin-1 neurons of the arcuate nucleus were activated through simultaneous administration of ghrelin and deacylated ghrelin. Probably, the deacylated ghrelin can inhibit the ghrelin sensitive NPY/AgRP neurons by acting through the nesfatin-1 releasing cells (Inhoff et al. 2008). It is worth noticing that presently, there is no experimental evidence suggesting any contribution of leptin to the nesfatin-1 anorexigenic mechanisms of action. The blockage of nesfatin-1-dependent signaling does not diminish leptin's physiological effects. In turn, leptin by itself does not appear to be a modulator of the NUCB2 and nesfatin-1 expression in hypothalamic nuclei (Oh et al. 2006, Garcia-Galiano et al. 2010). It has been known that the feeding suppression by leptin occurs via the POMC and CART neurons' activation, but it has not been reported that the nesfatin- 1 is capable of stimulation of this group of cells. This observation may confirm that nesfatin-1 has its own mode of action, which is leptin-independent (Shimizu et al. 2009). Nevertheless, there is some basis to consider that the anorexigenic action of nesfatin-1 was made possible through its influence on the hypothalamic melanocortin system. The key elements of this signaling pathway are the POMC neurons, which are a source of $\alpha-\mathrm{MSH}$, as well as PVN and SON cells, rich in $\mathrm{MC}_{3}$ and $\mathrm{MC}_{4}$ receptors (discussed in another subsection) (Oh et al. 2006, Yosten and Samson 2010).

A great majority (above 75\%) of nesfatin neurons located in the tuberal part of the hypothalamus reveals coexpression with melanin concentrating hormone (MCH). Therefore, one may suppose that nesfatin-1 can be involved in a number of other $\mathrm{MCH}$ dependent hypothalamic functions such as regulation of autonomic processes, stress response, mood, cognitive functions and sleep (Fort et al. 2008). 
2.2 Interactions with oxytocin, CRF and melanocortin pathway

Expression of nesfatin-1 is also manifested by numerous oxytocinergic and vasopressinergic perikarya of the paraventricular nucleus (PVN) and supraoptic nucleus (SON). Concentration of nesfatin-1 and the level of expression of NUCB2 in these nuclei decreases after 24 days of starvation. Refeeding results in a repeated activation of those cells, and is expressed by elevation of c-Fos levels (Kohno et al. 2008). Relatively large (on average 43\%) group of nesfatin PVN neurons reveals oxytocin expression, but above $40 \%$ of oxytocinergic neurons synthesize nesfatin-1 (Stengel et al. 2010a). This cytophysiological correlation allows us to suspect that there are some subtle functional associations between these two active hypothalamic peptides. This is confirmed by an observation that the action of ornithine vasotocin (OVT), an antagonist of the oxytocinergic receptor, relieves the anorexigenic effects of nesfatin-1 in rats. Nesfatin1 is a factor that significantly stimulates oxytocin secretion by magno- and parvocellular neurons (PVN) in rat. However, it has not been found that it causes the elevation of oxytocin concentration in serum (Yosten and Samson 2010). On the other hand, an increase of c-Fos expression in parvocellular neurons (PVN) has been noted.

There are some reports that the axons of parvocellular oxytocinergic neurons (PVN) arrive to the nucleus of solitary tract (NST), an integration area of central and peripheral pathways of food intake control, where they exert their inhibitory effect (Blevins et al. 2004, Sabatier 2006). Above $50 \%$ of PVN nesfatin cells synthesizes vasopressin, and about $23 \%$ of them thyreotropin releasing hormone (TRH). Functions of this cell population as yet have not been explained(Stengel et al. 2010a). A small (on average $19 \%)$ subpopulation of nesfatin PVN neurons display coexpression of CRF, a factor, which inhibits food intake in stressful circumstances, and thus, is suspected to play an important role in the pathogenesis of eating disorders (Stengel et al., 2010, Connan et al. 2007). Also, this group of cells has been considered to participate in generating strongly anorexigenic effects. Satiety, caused by intracerebroventricular (i.v.) 
administration of nesfatin-1 is relieved by administration of the CRF2 receptor antagonist - astressin 2 -B (Yosten and Samson 2010). It has also been noted that the injection of $\alpha-\mathrm{MSH}$ to the rat cerebral ventricles increases the expression of NUCB2 mRNA, a protein precursor of nesfatin-1 in the PVN neurons. This suggests that the cells which synthesize this peptide, act through the melanocortin receptors (Maejima et al. 2009)Although the mechanisms of these actions are still unknown, the veracity of the proposed hypothesis is supported by the fact that the change of NUCB2 expression levels had not been reported after prior use of SHU9119, a selective antagonist of melanocortin $\mathrm{MC}_{3}$ and $\mathrm{MC}_{4}$ receptors (Brailoiu et al. 2007). The melanocortin $\mathrm{MC}_{4}$ receptor in PVN plays a crucial role in the regulation of eating process, and therefore, one may speculate that the nesfatin-1 neurons, displaying coexpression of oxytocin, vasopressin, and CRF are the effectors in melanocortin signalization pathway (Kishi et al. 2003, Yosten and Samson 2009). It has also been reported that upon refeeding c-Fos expression in abdominal neurons of parvocellular PVN layer depends on the melanocortin signaling (Singru et al. 2007). One probable explanation may be that the initiation of feeding, after a period of starvation, affects the POMC neurons, which induce c-Fos expression in nesfatin-1 hypothalamic neurons. In addition, neuromedin U (NMU) can be regulated by nesfatin-1, because after its i.v. injection, the increase of cFos expression occurs in PVN and SON (Ozaki et al. 2002). It is worth noticing that one of the factors that activates nesfatin PVN and NTS neurons is an anorectic intestinal hormone cholecystokinin CCK-8S (Noetzel et al. 2009). A potential participation of nesfatin-1 in the gut-hypothalamus regulatory axis is supported by recent reports that abdominal surgery activates nesfatin-1 hypothalamic neurons (SON and PVN) of the rat (Stengel et al. 2010b). In contrast, an intraperitoneal injection of this peptide reduces the stomach emptying in rats (Stengel et al. 2009). In turn, an i.v. administration of nesfatin-1 inhibits gastroduodenal motility in mice (Atsuchi et al. 2010) 
3. Involvement of nesfatin-1 in the regulation of reproductive processes

A multilevel regulatory activity of autonomic centres and neuronal pathways is a noteworthy characteristic of many hypothalamic structures. The same groups of neurons are simultaneously involved in the performance of many regulatory functions. The aforementioned neurons of the hypothalamic nuclei: ARC, PVN, SON and LHA are responsible for maintenance of energetic and osmotic homeostasis. They are also involved in the central regulation of reproductive processes, including sexual maturation and mating behaviour. Expression of nesfatin-1 in these nuclei suggests its contribution to these physiological activities. The newest research studies seem to confirm these presumptions, and have found that nesfatin-1 expression in the rat hypothalamic nuclei increases significantly during the course of sexual maturation, and then achieves its peak between the 20-th and 35-th day of life. At that time, a 3-fold increase in the total amount of neuropeptide protein in PVN, SON and LHA occurs (Garcia-Galiano et al. 2010). It should be emphasized that this effect can be greatly disturbed by negative energy balance, caused by a short-term starvation or nutritional deficiency. It has also been noted that the intracerebroventricular administration of nesfatin-1 induces a significant, above 2-fold, increase in the LH concentration in the serum of female rats, being fed in a standard way. In contrast, the short-term starvation causes a dramatic increase of the gonadotropin responses to nesfatin-1. A 9-fold increase in the LH level, within 15 minutes after an injection has been reported (Garcia-Galiano et al. 2010). During puberty, a functional blockage of nesfatin-1 in hypothalamus delays an appearance of external sexual signs (e.g.: vaginal opening), causes a decrease of the ovarian mass, and a reduction of the LH serum concentration. Thus, some valid presumptions exist that nesfatin-1 can also participate in the regulation of gonadotropin secretion in adulthood. Despite some preliminary studies, which have not revealed any 
statistically significant differences in the gonadotropin levels among adult female rats exposed to the central action of nesfatin-1 (Garcia-Galiano et al. 2010), some reports have appeared, suggesting that the high doses of this neuropeptide can modulate the LH and FSH concentrations in male adult rats (Tadross et al. 2010). Although full explanation of this problem requires further studies, even at the present stage of knowledge, nesfatin-1 can be considered as one of the regulatory factors of the hypothalamic-pituitary-axis. Some signaling processes, in which it is involved, make an important contribution to the gonadotropin secretion during puberty. It should also be underlined that these processes are downregulated by distorted energy homeostasis.

4. Neuropsychiatric aspects of nesfatin-1 physiological actions

\subsection{Stress responses and depression}

Many of the recent studies suggest that acute restrain stress is one of the factors activating nesfatin PVN, SON, NTS and EW neurons (Stengel et al. 2010a, Goebel et al. 2009) (Fig 3.). Total adrenalectomy leads to an increase of NUCB2 mRNA expression in PVN, and i.v. nesfatin-1 injection causes an elevation of the stress hormones: ACTH and corticosterone in the serum (Könczöl et al. 2010). Nesfatin-1 seems to contribute to generalized signs of stress. Administration of this peptide into lateral ventricles of the rat brain causes an elevation of blood pressure (Yosten et Samson. 2009). It has been suggested that the hypertensive effects of centrally administered nesfatin-1 is also the result of stimulation of renal sympathetic nerves, mediated via melanocortin hypothalamic pathways (Tanida and Mori 2011). In addition, it has been noted that the expression of nesfatin-1 in raphe nuclei, LC and EW neurons, in rats exposed to action of different stressors such as wrap restraint stress (Okere et al. 2010, Goebel et al. 2009,), abdominal surgery (Stengel et al. 2010b) and lipopolysaccharide administration was 
increased (Bonnet et al. 2009). Nesfatin-1 activates stress-sensitive serotoninergic neurons of raphe nuclei, and noradrenergic LC neurons, which in turn stimulate the CRF neurons in PVN, and then activate the HPA axis (Yoshida et al. 2010). It has been known that the raphe nuclei and LC are also the key centres of the serotoninergic and noradrenergic brain signaling system. Their dysfunctions are closely correlated with pathogenesis of depression and anxiety disorders. At the present time, it seems probable that nesfatin-1 can play a hypothetical and nonspecific role in some of these mechanisms. Some authors suggest that nesfatin-1 induces anxiety or fear reactions (Merali et al. 2008, Oh et al. 2006) and perhaps depressive reactions (Chaki and Okubo 2007), via activation of melanocortin pathways. This results in inhibition of GABA-ergic neurons or alternatively, in hyperpolarization of NPY neurons in the arcuate nucleus (Domschke et al. 2010). In patients with major depressive disorder (MDD), a higher serum nesfatin-1 level has been revealed, comparing to the one reported in the normal population (Ari et al. 2011). In general, these results support bidirectional permeability of blood-brain barrier for nesfatin-1. However, at the present moment, these findings are still difficult to interpret. This is due to lack of information, indicating which neuronal cell populations of the brain are responsible for the increased nesfatin-1 secretion in patients with MDD. The underlying mechanism of this phenomenon is still unclear. Also, it cannot be excluded that the additional source of circulating nesfatin-1 may be secondarily activated by some cells, located outside of the CNS. An intriguing study performed recently by Bloem et al. (2011) has shown that nesfatin-1/NUCB2 mRNA expression in the human Westphal-Edinger nucleus (EW) was higher in suicidal cases among males (all victims did not have previously diagnosed psychiatric or neurodegenerative disorders), whereas among females, this content was lower, compared to controls. The authors have also found nesfatin-1/NUCB2 and CART colocalization in the EW, and they were the first to show sex-related changes in the neuropeptides levels in the brain stem of suicide victims versus controls. These findings may suggest the existence of nesfatin-CART interplay in the midbrain. However, due to lack of other evidence on this topic, they are still difficult to interpret. Undoubtedly, the 
potential role of nesfatin-1 signaling in the pathogenesis of depressive-like behaviour requires further study.

\subsection{Eating disorders}

Due to some anorexigenic properties of nesfatin-1, it seems justified to conduct further research studies analyzing its potential role in pathogenesis of psychogenic eating disorders. Recently, it has been noted that plasma nesfatin-1 levels in patients suffering from restricting-type anorexia nervosa (AN-R) were significantly lower, compared with healthy persons. This may indicate a negative correlation with ghrelin and des-acyl ghrelin levels. In contrast, a positive correlation between nesfatin-1 levels and BMI was demonstrated (Ogiso et al. 2011). An opposite phenomenon was displayed in healthy men, with normal body mass index, in whom the fasting nesfatin-1 concentration negatively correlated with their BMI (Tsuchiya et al. 2010). This observation was similar to the one reported in rats (Stengel et al. 2009). However, there is still no convincing evidence that the low nesfatin-1 level underlies anxiety disorders, often accompanying AN-R. On the other hand, it cannot be excluded that during periods of extreme starvation, even the decreased nesfatin-1 level may reduce anxiety or fear, and stimulate food-intake. Unfortunately, a very limited number of research papers on this topic does not allow us to precisely interpret these phenomena. It appears that some valuable data, contributing to this discussion, could be provided by brain imaging studies (PET, SPECT), using radiolabelled nesfatin-1. 


\subsection{Epilepsy}

The initial interesting reports regarding correlations between nesfatin-1 levels in body fluids and a course of epilepsy were published by Aydin et al. in 2009. It was noted that the primary generalized epilepsy (PGE) and secondary generalized seizures (SGE) significantly elevated nesfatin-1 levels in the patients' plasma and saliva. During treatment, there was a decrease of this neuropeptide concentration, but nevertheless, it did not reach the level which is typical for healthy individuals. The newest studies, conducted by the same authors support the previous data. They also indicate that the plasma nesfatin-1 level is in the highest range during the 5 minutes after onset of the PGE and SGE attack. After that, it decreases significantly, and then, after 48 hours, it decreases to approximately $50 \%$ of its original level (Aydin et al. 2011). However, the exact mechanisms of these changes have not been clearly explained. Some hypotheses have been formulated that the elevated nesfatin-1 level can induce some excitotoxic effects that may disturb certain activities of cortical and subcortical neuronal circuitries. This may result in onset of epileptic symptoms.

It has also been found that the plasma nesfatin-1 level was significantly elevated in rats with epileptic seizures, induced by kainic acid. This suggests that the plasma level of this neuropeptide may illustrate a profile of its fluctuation in hypothalamus. (Liu et al. 2011) Although this issue certainly requires further study, at the present time, nesfatin-1 can be considered as a valuable and promising marker of epileptic episodes, being potentially useful in a neurological diagnostic work-up. 


\section{Concluding remarks}

Nesfatin-1 is a neuropeptide characterized by a wide spectrum of activity in the CNS. There is some evidence to consider nesfatin-1 as a promising factor which can be useful for clinical application. It can be potentially helpful in the therapy of metabolic disorders, and obesity of various origins. Elevated plasma nesfatin-1 levels in patients suffering from primary generalized epileptic seizures, suggests its potential use as a biomarker in epilepsy diagnostic work-up (Aydin et al. 2009). Increase in body mass and BMI among psychiatric patients taking antipsychotic, antidepressive, and other psychotropic medications represents a huge clinical problem. In many of these obese patients, a substantial reduction of sensitivity to leptin or some other adipokines may occur, in various hypothalamic centres (Haymsfield et al. 1999). It seems that in these cases, the use of nesfatin-1 can cause a substantial clinical improvement. Based on experimental studies, an intraperitoneal administration of nesfatin-1 has significantly reduced food intake in leptin-resistant animals. In addition, nesfatin-1 has been found to reduce an elevated body mass of experimental animals. A possible relationship between nesfatin-1 action and appetite regulation in patients suffering from chronic kidney disesase (CKD) has also been cautiously postulated (Saldanha et al. 2011).

One possible way of administration of this agent is a subcutaneous injection. However, a particularly interesting and beneficial therapeutic view seems to be its intranasal administration. It has been effective in some experimental animal models (Shimizu et al. 2009). Although the results of many experimental studies are encouraging, any possible clinical applications of nesfatin-1 still remain in the area of speculation. Further research on this topic certainly merits attention. In the mean time, answers to several questions, concerning pharmacokinetics, potential adverse drug reactions, tolerability profile and interactions with other medications are required. Another potential research direction consists of searching for selective nesfatin-1 antagonists that perhaps can contribute to opening of a new chapter in therapy of eating disorders such as anorexia nervosa. However it should be emphasized that the nesfatin-1 receptor so far remains uncloned. 
Therefore, the further progress of pharmacological studies in this field is still very limited.

\section{Acknowledgement}

The authors would particularly like to acknowledge the contribution of Kate Rygiel, MD to the preparation of this article.

\section{REFERENCES}

1. Ari M., Ozturk O.H. Bez Y., Oktar S., Erduran D., 2011. High plasma nesfatin-1 level in patients with major depressive disorder. Prog Neuropsychopharmacol Biol Psychiatry 35, 497-500.

2. Atsuchi K., Asakawa A., Ushikai M., Ataka K., Tsai M., Koyama K., Sato Y., Kato I., Fujimiya M., Inui A., 2010. Centrally administered nesfatin-1 inhibits feeding behaviour and gastroduodenal motility in mice. Neuroreport 21, 1008-1011.

3. Aydin S., Dag E., Ozkan Y., Arslan O., Koc G., Bek S., Kirbas S., Kasikci T., Abasli D., Gokcil Z., Odabasi Z., Catak Z., 2011. Time-dependent changes in the serum levels of prolactin, nesfatin-1 and ghrelin as a marker of epileptic attacks in young male patients. Peptides, 32, 1276-1280.

4. Aydin S., Dag E., Ozkan Y., Erman F., Dagli A.F., Kilic N., Sahin I., Karatas F., Yoldas T., Barim A.O., Kendir Y., 2009. Nesfatin-1 and ghrelin levels in serum and saliva of epileptic patients: hormonal changes can have a major effect on seizure disorders. Mol Cell Biochem 328, 49-56.

5. Blevins J.E., Schwartz M.W., Baskin D.G., 2004. Evidence that paraventricular nucleus oxytocin neurons link hypothalamic leptin action to caudal brain stem nuclei controlling meal size. Am J Physiol Regul Integr Comp Physiol 287, R87-96.

6. Bloem B., Xu L., Morava E., Faludi G., Palkovits M., Roubos E.W., Kozicz T., 2011. Sex-specific differences in the dynamics of cocaine and amphetamine-regulated transcript and nesfatin- 1 expressions in the midbrain of depressed suicide victims vs. controls. Neuropharmacology doi:10.1016/j.neuropharm.2011.07.023. 
7. Bonnet M.S., Pecchi E., Trouslard J., Jean A., Dallaporta M., Troadec J.D., 2009. Central nesfatin-1 expressing neurons are sensitive to peripheral imflammatory stimulus. J Neuroinflammation 6, 27.

8. Brailoiu G.C., Dun S.L., Brailoiu E., Inan S., Yang J., Chang J.K., Dun N.J., 2007. Nesfatin-1: distribution and interaction with a $\mathrm{G}$ protein-coupled receptor in the rat brain. Endocrinology 148, 5088-5094.

9. Chaki S., Okubo T., 2007. Melanocortin-4 receptor antagonists for the treatment of depression and anxiety disorders. Curr Top Med Chem 7, 1145-1151.

10. Connan F., Lightman S.L., Landau S., Wheeler M., Treasure J., Campbell I.C.,2007. An investigation of hypothalamic-pituitary-adrenal axis hyperactivity in anorexia nervosa: the role of CRH and AVP. J Psychiatr Res 41, 131-143.

11. Domschke K., Danniowski U., Hohoff C., Ohrmann P., Bauer J., Kugel H., Zwanger P., Heindel W., Deckert J., Arolt V., Suslow T., Baune B.T., 2010. Neuropeptide Y (NPY) gene: Impact on emotional processing and treatment response in anxious depression. Eur Neuropsychopharmacol 20, 301-309.

12. Foo K.S., Brismar H, Broberger C., 2008. Distribution and neuropeptide coexistence of nucleobindin-2 mRNA/nesfatin-like immunoreactivity in the rat CNS. Neuroscience 156, 563-579.

13. Fort P., Salvert D., Hanriot L., Jego S., Shimizu H., Hashimoto K., Mori M., Luppi PH., 2008. The satiety molecule nesfatin-1 is co-expressed with melanin concentrating hormone in tuberal hypothalamic neurons of the rat. Neuroscience 155 , 174-181.

14. Garcia-Galiano D., Navarro V.M., Roa J., Ruiz-Pino F., Sanchez-Garrido M.A., Pineda R., Castellano J.M., Romero M., Aguilar E., Gaytan F., Dieguez C., Pinilla L., Tena-Sempere M., 2010. The anorexigenic neuropeptide, nesfatin-1, is indispensable for normal puberty onset in the female rat. J Neurosci 30, 7783-7792.

15. Garcia-Galiano D., Navarro V.M., Gaytan F., Tena-Sempere M., 2010a. Expanding roles of NUCB2/nesfatin-1 in neuroendocrine regulation. J Mol Endocrinol 45, 281290.

16. Goebel M., Stengel A., Wang L., Lambrecht N.W., Taché Y., 2009. Nesfatin-1 immunoreactivity in rat brain and spinal cord autonomic nuclei. Neurosci Lett 452, 241-246.

17. Goebel-Stengel M., Wang L., Stengel A., Tache Y., 2011. Localization of nesfatin-1 neurons in the mouse brain and functional implication. Brain Res 1396, 20-34.

18. Gonzalez R., Reingold B.K., Gao X., Gaidhu M.P., Tsushima R.G., Uniappan S., 2011. Nesfatin-1 exerts a direct, glucose-dependent insulinotropic action on mouse islet $\beta$ - and MIN6 cells. J Endocrinol 208, R9-R16. 
19. Gonzalez R., Kerbel B., Chun A., Uniappan S., 2010. Molecular, cellular and physiological evidences for anorexigenic actions of nesfatin-1 in goldfish. PLoS One 5, e15201.

20. Heymsfield S.B., Greenberg A.S., Fujioka K., Dixon R.M., Kushner R., Hunt T., Lubina J.A., Patane J., Self B., Hunt P., McCamish M., 1999. Recombinant leptin for weight loss in obese and lean adults: a randomized, controlled, dose-escalation trial. JAMA 282, 1568-1575.

21. Inhoff T., Stengel A., Peter L., Goebel M., Taché Y., Bannert N., Wiedenmann B., Klapp B.F., Mönnikes H., Kobelt P., 2010. Novel insight in distribution of nesfatin-1 and phospho-mTOR in the arcuate nucleus of the hypothalamus of rats. Peptides 31 , 257-262.

22. Inhoff T., Mönnikes H., Noetzel S., Stengel A., Goebel M., Dinh Q.T., Riedl A., Bannert N., Wisser A.S., Wiedenmann B., Klapp B.F., Taché Y., Kobelt P., 2008. Desacyl ghrelin inhibits the orexigenic effect of peripherally injected ghrelin in rats. Peptides 29, 2159-2168.

23. Iwasaki Y., Nakabayashi H., Kakei M., Shimizu H., Mori M., Yada T, 2009. Nesfatin-1 evokes Ca2+ signaling in isolated vagal afferent neurons via $\mathrm{Ca} 2+$ influx through N-type channels. Biochem Biophys Res Commun 390, 958-962.

24. Liu Z., Wang F., Li Z.Z., Qi J.H., Xu W.Z., Zhang P.S., Sun T., 2011. Expression of neuropeptides ghrelin and nesfatin-1 in kainic acid kindling rats. Zhonghua Yi Xue Za Zhi 91, 496-500.

25. Kishi T., Aschkenasi C.J., Lee C.E., Mountjoy K.G., Saper C.B., Elmquist J.K., 2003. Expression of melanocortin 4 receptor mRNA in the central nervous system of the rat. J Comp Neurol 457, 213-235.

26. Kohno D., Nakata M., Maejima Y., Shimizu H., Sedbazar U., Yoshida N., Dezaki K., Onaka T., Mori M., Yada T., 2008. Nesfatin-1 neurons in paraventricular and supraoptic nuclei of the rat hypothalamus coexpress oxytocin and vasopressin and are activated by refeeding. Endocrinology 149, 1295-1301.

27. Könczöl K., Bodnar I., Zelena D., Pinter O., Papp R.S., Palkovits M., Nagy G.M., Toth Z.E., 2010. Nesfatin-1/NUCB2 may participate in the activation of the hypothalamic-pituitary-adrenal axis in rats. Neurochem Int 53, 189-197.

28. Maejima Y., Sedbazar U., Suyama S., Kohno D., Onaka T., Takano E., Yoshida N., Koike M., Uchiyama Y., Fujiwara K., Yashiro T., Horvath T.L., Dietrich M.O., Tanaka S., Dezaki K., Oh-I S., Hashimoto K., Shimizu H., Nakata M., Mori M., Yada T., 2009. Nesfatin-1-regulated oxytocinergic signaling in the paraventricular nucleus causes anorexia through a leptin-independent melanocortin pathway. Cell Metab 10, 355-365.

29. Merali Z., Cayer C., Kent P., Anisman H., 2008. Nesfatin-1 increases anxiety- and fear-related behaviors in the rat. Psychopharmacology (Berl) 201, 115-123. 
30. Nakata M., Manaka K., Yamamoto S., Mori M., Yada T., 2011. Nesfatin-1 enhances glucose-induced insulin secretion by promoting $\mathrm{Ca}^{2+}$ influx through L-type channels in mouse b-cells. Endocr J 58, 305-313.

31. Noetzel S., Stengel A., Inhoff T., Goebel M., Wisser A.S., Bannert N., Wiedenmann B., Klapp B.F., Tache Y., Mönnikes H., Kobelt P., 2009. CCK-8S activates c-Fos in a dose-dependent manner in nesfatin-1 immunoreactive neurons in the paraventricular nucleus of the hypothalamus and in the nucleus of the solitary tract in the brain stem. Regul Pept 157, 84-91.

32. Oh I. S., Shimizu H., Satoh T., Okada S., Adachi S., Inoue K., Eguchi H., Yamamoto M., Imaki T., Hashimoto K., Tsuchiya T., Monden T., Horiguchi K., Yamada M., Mori M., 2006. Identification of nesfatin-1 as a satiety molecule in the hypothalamus. Nature 443, 709-712.

33. Ogiso K., Asakawa A., Amitani H., Nakahara T., Ushikai M., Haruta I., Koyama KI. Amitani M., Harada T., Yasuhara D., 2011. Inui A. Plasma nesfatin-1 concentrations in restricting-type anorexia nervosa. Peptides 32, 150-153.

34. Okere B., Xu L., Roubos E.W., Sonetti D., Kozicz T., 2010. Restraint stress alters the secretory activity of neurons co-expressing urocortin-1, cocaine- and amphetamine-regulated transcript peptide and nesfatin-1 in the mouse EdingerWestphal nucleus. Brain Res 1317, 92-99.

35. Ozaki Y., Onaka T., Nakazato M., Saito J., Kanemoto K., Matsumoto T., Ueta Y., 2002. Centrally administered neuromedin $U$ activates neurosecretion and induction of c-fos messenger ribonucleic acid in the paraventricular and supraoptic nuclei of rat. Endocrinology 143, 4320-4329.

36. Özsavci D., Ersahin M., Sener A., Özakpinar Ö.B., Toklu H.Z., Akakin D., Sener G., Yegen B.C., 2011. The novel function of nesfatin-1 as an anti-inflammatory and antiapoptotic peptide in subarachnoid hemorrhage-induced oxidative brain damage in rats. Neurosurgery 68, 1699-1708.

37. Pałasz A., Bryzek A., Krzystanek M., Wiaderkiewicz R., 2010. Anoreksygenna aktywnosc nesfatyny-1 w jadrach podwzgórza i jej potencjalne implikacje kliniczne/Anorexigenic activity of nesfatin-1 in hypothalamic nuclei and its potential clinical implications. Farmakoter Psychiatr Neurol 26, 39-43.

38. Pan W., Hsuchou H., Kastin A.J., 2007. Nesfatin-1 crosses the blood-brain barrier without saturation. Peptides 28, 2223-2228.

39. Price C.J., Samson W.K., 2008. Ferguson A.V., Nesfatin-1 inhibits NPY neurons in the arcuate nucleus. Brain Res 1230, 99-106.

40. Ramanjaneya M., Chen J., Brown J.E., Tripathi G., Hallschmid M., Patel S., Kern W., Hillhouse E.W., Lehnert H., Tan B.K., Randeve H.S., 2010. Identification of nesfatin-1 in human and murine adipose tissue: a novel depot-specific adipokine with increased levels in obesity. Endocrinology 151, 3169-3180. 
41. Sabatier N., 2006. alpha-Melanocyte-stimulating hormone and oxytocin: a peptide signalling cascade in the hypothalamus. J Neuroendocrinol 18, 703-710.

42. Saldanha J.F., Carrero J.J., Mafra D., 2011. The possible role of nesfatin-1 on appetite regulation in hemodialysis patients. Med Hypotheses doi:10.1016/j.mehy.2011.07.006

43. Stengel A., Goebel M., Wang L., Rivier J., Kobelt P., Mönnikes H., Lambrecht N.W., Taché Y., 2009. Central nesfatin-1 reduces dark-phase food intake and gastric emptying in rats: differential role of corticotropin-releasing factor 2 receptor. Endocrinology 150, 4911-9.

44. Stengel A., Goebel M., Wang L., Tache Y., 2010. Ghrelin, des-acyl ghrelin and nesfatin-1 in gastric X/A-like cells: role as regulators of food intake and body weight. Peptides 31, 357-369.

45. Stengel A., Goebel M., Tache Y., 2010a. Nesfatin-1: a novel inhibitory regulator of food intake and body weight. Obesity Rev 12, 261-271.

46. Stengel A., Goebel M., Wang L., Tache Y., 2010b. Abdominal surgery activates nesfatin-1 immunoreactive brain nuclei in rats. Peptides 31, 263-270.

47. Stengel A., Tache Y., 2010. Nesfatin-1: role as a possible new potent regulator of food intake. Regul Pept 9, 18-23.

48. Stengel A., Tache Y., 2011. Minireview: Nesfatin-1 an emerging new player in the brain-gut, endocrine and metabolic axis. Endocrinology, DOI:. 10.1210/en.20111500

49. Shimizu H., Oh.I. S., Okada S., Mori M., 2009. Nesfatin-1: an overview and future clinical application. Endocr J 56, 537-543.

50. Singru P.S., Sánchez E., Fekete C., Lechan R.M., 2007. Importance of melanocortin signaling in refeeding-induced neuronal activation and satiety. Endocrinology 148, 638-646.

51. Su Y., Zhang J., Tang Y., Bi F., Liu J.N., 2010. The novel function of nesfatin-1: anti-hyperglycemia. Biochem Biophys Res Commun 391, 1039-1042.

52. Tadross J.A., Patterson M., Wynne K.J., Patel S., Suzuki K., Ghatei M.A., Bloom S.R., 2010. Nesfatin supresses feeding and stimulates the hypothalamo-pituitarygonadal axis. Endocrine J 57, S355-S648.

53. Tan B.K., Hallschmid M., Kern W., Lehnert H., Randeva H.S., 2011. Decreased cerebrospinal fluid/plasma ratio of the novel satiety molecule, nesfatin-1/NUCB-2 in obese humans: evidence of nesfatin-1/NUCB-2 resistance and implications for obesity treatment. J Clin Endocrinol Metab 96, E669-E673.

54. Tanida M., Mori M., 2011. Nesfatin-1 stimulates renal sympathetic nerve activity in rats. Neuroreport 22, 309-312. 
55. Tsuchiya T., Shimizu H., Yamada M., Osaki A., Oh I.S., Ariyama Y., Takahashi H., Okada S., Hashimoto K., Satoh T., Kojima M., Mori M., 2010. Fasting concentrations of nesfatin-1 are negatively correlated with body mass index in nonobese males. Clin Endocrinol 73, 484-490.

56. Yoshida N., Maejima Y., Sedbazar U., Ando A., Kurita H., Damdindorj B., Takano E., Gantulga D., Iwasaki Y., Kurashina T., Onaka T., Dezaki K., Nakata M., Mori M., Yada T., 2010. Stressor-responsive central nesfatin-1 activates corticotropinreleasing hormone, noradrenaline and serotonin neurons and evokes hypothalamicpituitary-adrenal axis. Aging 2, 775-784.

57. Yosten G.L.C., Samson W.K., 2009. Nesfatin-1 exerts cardiovascular actions in brain: possible interaction with the central melanocortin system. Am J Physiol Regul Integr Comp Physiol 297, R1330-R1336.

58. Yosten G.L.C., Samson W.K., 2010. The anorexigenic and hypertensive effects of nesfatin-1 are reversed by pretreatment with an oxytocin receptor antagonist. Am J Physiol Regul Integr Comp Physiol 298, R1642-R1647.

59. Zhang A.Q., Li X.L., Jiang C.Y., Lin L., Shi R.H., Chen J.D., Oomura Y., 2010. Expression of nesfatin-1/NUCB2 in rodent digestive system. World J Gastroenterol $16,1735-1741$. 


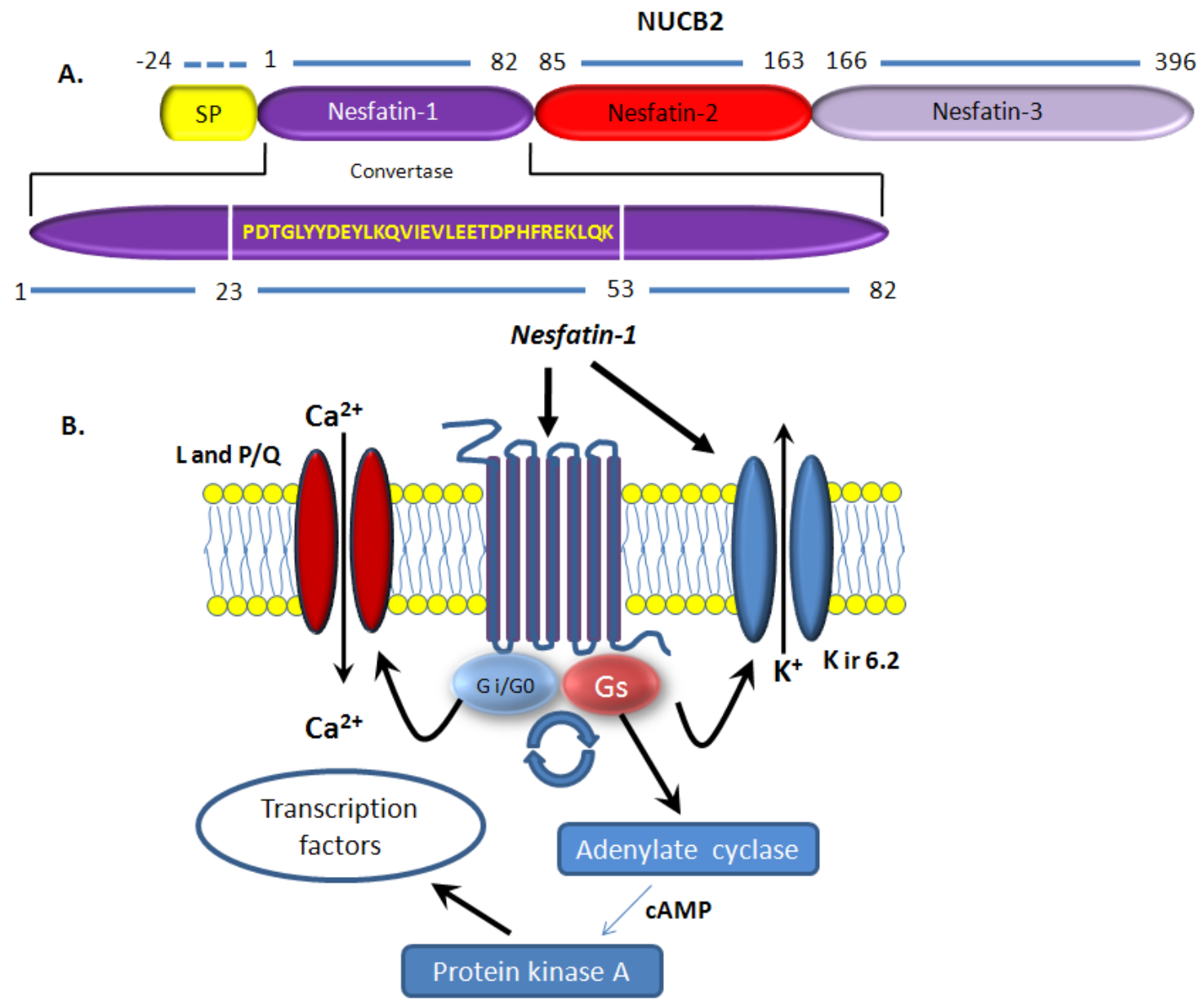

Fig. 1. The primary structure of nesfatin-1 and its prohormone NUCB2. (A) SP, signal peptide. Nesfatin-1 interaction with a putative G-coupled receptor leads to opening of the calcium channels ( $\mathrm{L}$ and $\mathrm{P} / \mathrm{Q}$ ) or/and to activating of the adenylate cyclase pathway and protein kinase $A$ in the hypothalamic neurons. The neuropeptide may also inhibit the NPY/AgRP cells directly, causing their hyperpolarization, through the ATP-dependent potassium channels $\mathrm{K}_{\text {ir6.2. }}(\mathrm{B})$. 


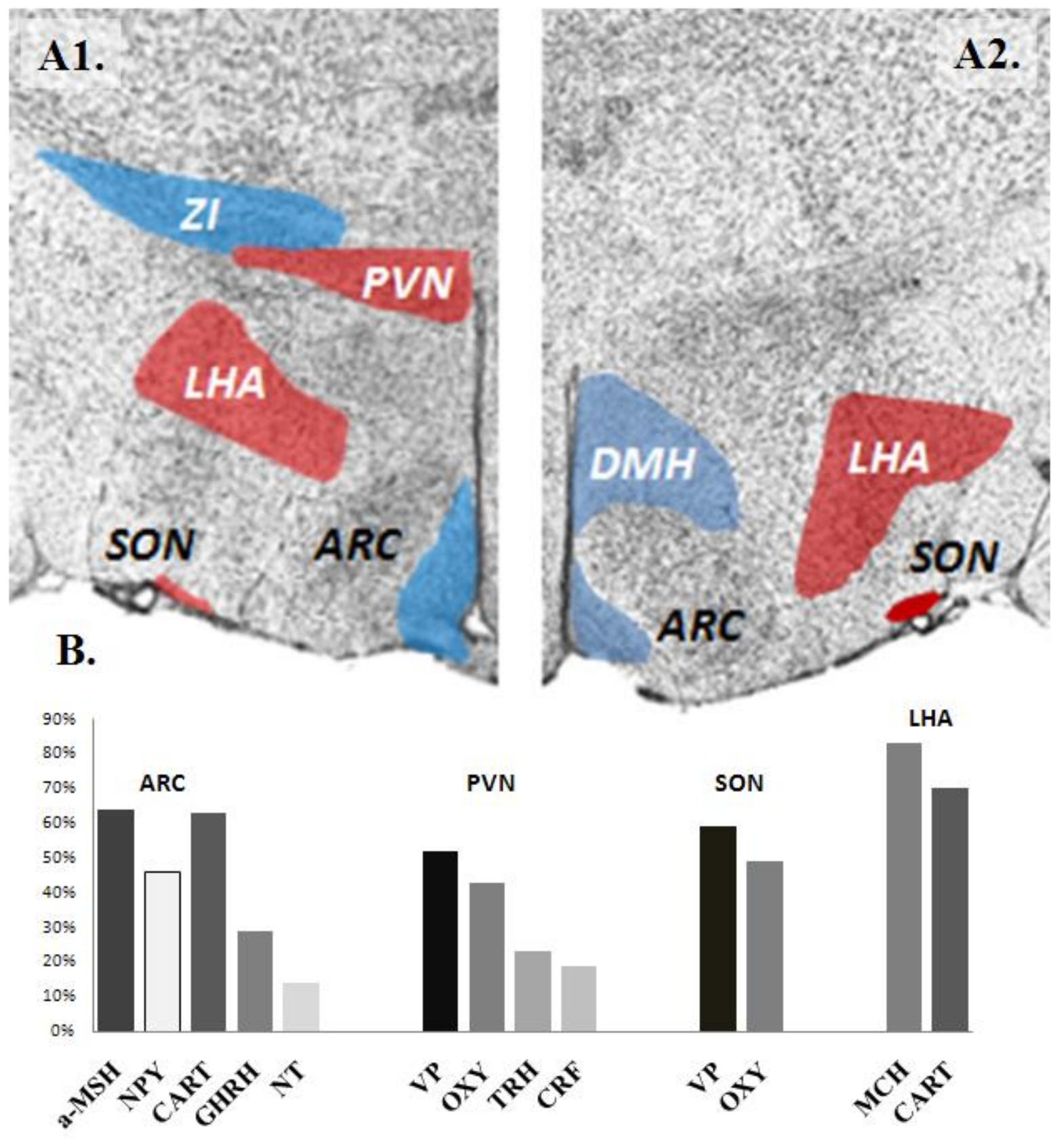

Fig 2. Schematic representation of nesfatin-1 neuronanatomical distribution in the rat hypothalamus. (A) ARC, arcuate nucleus; $\mathrm{DMH}$, dorsomedial hypothalmus; LHA, lateral hypothalamus; PVN, paraventricular nucleus; SON, supraoptic nucleus; ZI, zona incerta. Rat brain coronal sections according to Paxinos G., Watson C.; Rat Brain Atlas (2007); $2.04 \mathrm{~mm}$ (A1) and $-2.52 \mathrm{~mm}$ (A2) from bregma. The highest level of nesfatin-1 immunoreactivity was detected at the LHA, PVN, SON (red areas) and fainter reaction was observed at the $\mathrm{ZI}, \mathrm{ARC}$ and $\mathrm{DMH}$ (blue areas). Coexpression of nesfatin-1 immunoreactivity with other hypothalamic peptides (B). The graph shows the mean percentage of neurons positive for other transmitters colocalized with nesfatin-1. a$\mathrm{MSH}$, Melanocyte stimulating hormone $\alpha$; CRF, corticotropin releasing factor; CART, cocaine-amphetamine regulated transcript; $\mathrm{GHRH}$, growth hormone-releasing hormone; NT, neurotensin; OX oxytocin; TRH thyreotropin releasing hormone; VP, vasopressin. 


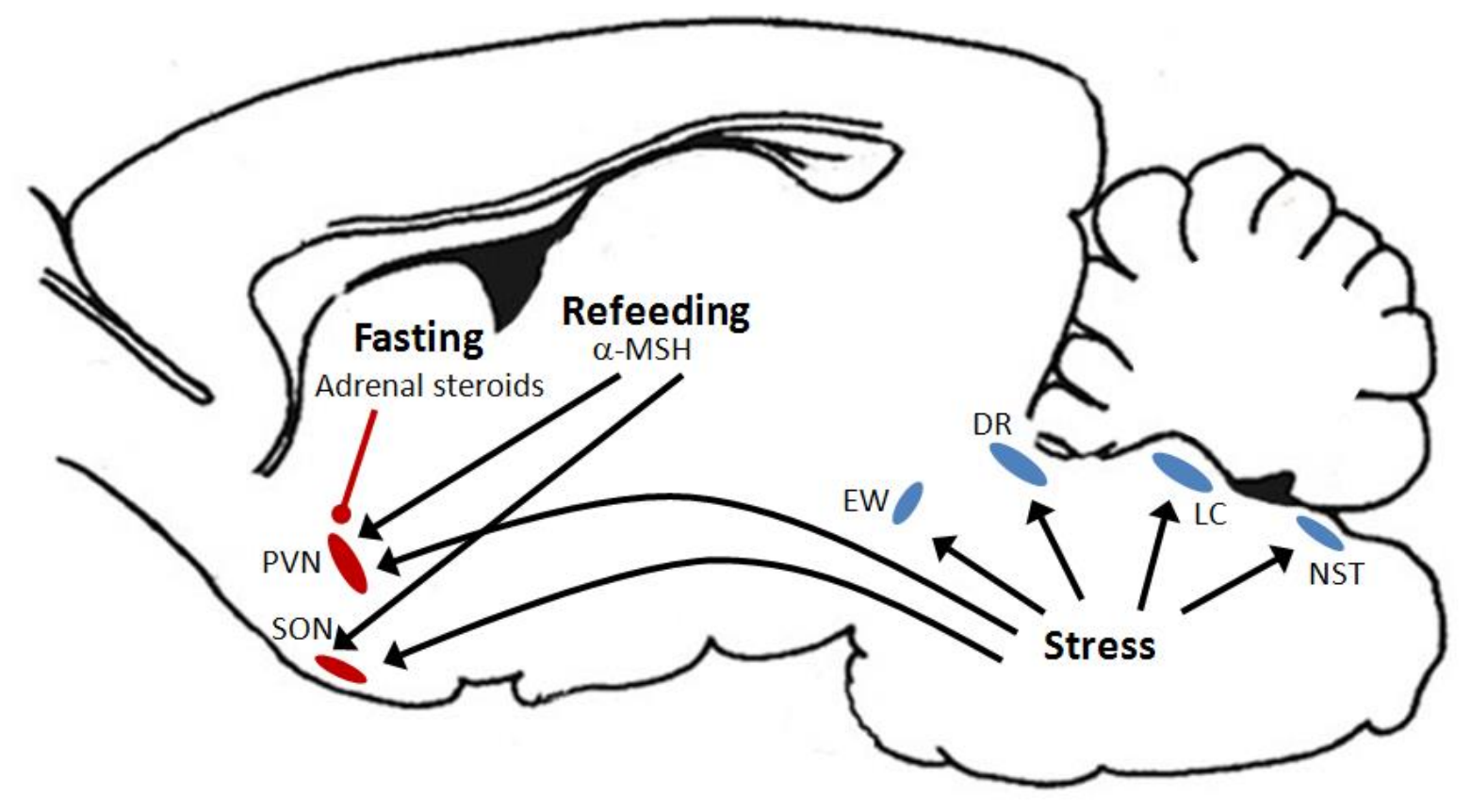

Fig. 3. An outline model of the stimulatory (black arrows) and inhibitory (round-ended line) regulation of nesfatin-1 signaling pathways in the rat brain. The picture emphasizes the important role of stress factors. DR, dorsal raphe; EW, Westphal-Edinger nucleus; LC, locus coeruleus; NTS, nucleus of the solitary tract; PVN, paraventricular nucleus; SON supraoptic nucleus. See text for further details. 